\title{
Studies on the copigmentation between anthocyanins and flavan-3-ols and their influence in the colour expression of red wine
}

\author{
Susana González-Manzano, Montserrat Dueñas, Julián C. Rivas-Gonzalo, \\ M. Teresa Escribano-Bailón, Celestino Santos-Buelga *

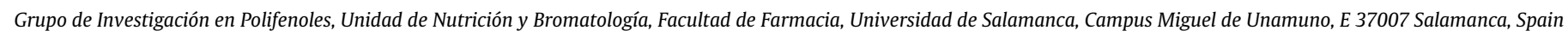

\section{A R T I C L E I N F O}

\section{Article history:}

Received 5 February 2008

Received in revised form 2 September 2008

Accepted 1 October 2008

\section{Keywords:}

Copigmentation

Catechins

Procyanidins

Red wine colour

CIELAB

Anthocyanin hydration constant

\begin{abstract}
A B S T R A C T
With the aim of evaluating the importance of the copigmentation process between anthocyanins and flavanols on the colour expression of red wine, assays were carried out in wine model systems with mixtures of compounds obtained from two Vitis vinifera grape varieties (Graciano and Tempranillo). Spectrophotometric and chromatic analyses were performed to evaluate the magnitude of the copigmentation and the modifications induced in the colour of the solutions. Measurement of the changes in the anthocyanin hydration constant $\left(K_{\mathrm{h}}\right)$ was also used to determine the strength of the copigmentation process. All the flavanols assayed induced significant changes in the colour, perceptible to the human eye, of the wine-like anthocyanin solutions at concentrations similar to those that can exist in red wines. The percentage contribution of the copigmentation with flavanols to the colour of the anthocyanin solutions was found to range from $2 \%$ to $20 \%$. The extent of this effect was related not only to the concentration of flavanols but also to the qualitative composition of the flavanol preparations, as influenced by the part of the grape (either skin or seed) and the variety considered. Divergences were found between the evaluation of the copigmentation based on chromatic parameters in the CIELAB colour space and that based on the measurement at visible $\lambda_{\max }$, as the latter does not consider the integral colour changes produced in the visible spectrum. The results obtained confirmed the importance of the qualitative phenolic composition, determined in the wine by the type of grape and winemaking practices, to the production of an effective copigmentation process.
\end{abstract}

(c) 2008 Elsevier Ltd. All rights reserved.

\section{Introduction}

Copigmentation is one of the most important factors leading to the profuse colour variability observed in flowers and fruits and it is also supposed to account for a variable part of the colour of red wines (Asen, Stewart, \& Norris, 1972; Timberlake \& Bridle, 1976). Copigmentation consists of hydrophobic interactions $(\pi-\pi$ stacking) between the planar polarisable nuclei of the coloured forms of the anthocyanins and other molecules (i.e., copigments). Copigmentation complexes adopt a sandwich configuration that protects the flavylium chromophore from the nucleophilic attack of water, thus reducing the formation of colourless hemiketal and chalcone forms. The final result is that anthocyanin solutions show a more intense colour than theoretically could be expected according to the $\mathrm{pH}$ value of the media (Goto \& Kondo, 1991). Different types of compounds have been evaluated as potential anthocyanin copigments, including alkaloids, amino acids, nucleotides, carbohydrates and phenolic compounds (Asen et al., 1972; Brouillard, Wigand, Dangles, \& Cheminat, 1991; Davis \& Mazza, 1993; Mistry, Cai, Lilley,

\footnotetext{
* Corresponding author. Tel.: +34 923294537; fax: +34 923294515

E-mail address: csb@usal.es (C. Santos-Buelga).
}

\& Haslam, 1991). In addition, anthocyanin chromophores may also associate among themselves (self-association), as well as with aromatic residues of their own molecule (intramolecular copigmentation). Copigmentation processes seem of critical importance in understanding the relationship between grape composition and colour in young red wines (Boulton, 2001) and they could also act as a first stage in the formation of new pigments that determine the colour of aged red wines (Brouillard \& Dangles, 1994).

Among red wine components, flavonoids and hydroxycinnamoyl derivatives appear to be most promising compounds to act as anthocyanin copigments. In assays carried out in wine model solutions, flavonols behave as powerful anthocyanin copigments, whereas flavan-3-ols were comparatively poor cofactors with hydroxycinnamic acids showing intermediate behaviour (Brouillard et al., 1991; Gómez-Míguez, González-Manzano, EscribanoBailón, Heredia, \& Santos-Buelga, 2006). The levels of these compounds are extremely variable in red wines, depending on the characteristics of the grape and the winemaking process. In general, it is assumed that the contents of flavonols and hydroxycinnamoyl derivatives are much lower than those of flavanols, which, as either monomers (catechins) or polymers (i.e., condensed tannins, proanthocyanidins), are major phenolic components in red wines. 
The comparatively small ability of flavanols to act as copigments is attributed to their non-planar structure that impedes a close approach to the anthocyanin and reduces the potential surface area available for hydrophobic stacking. However, important differences may exist among flavanols regarding conformation and efficiency as anthocyanin copigments. Among monomers, epicatechin is consider a better copigment than catechin (Brouillard et al., 1991; Liao, Cai, \& Haslam, 1992; Mirabel, Saucier, Guerra \& Glories, 1999 ), which is explained by the preferential quasi-equatorial conformation of its ring $B$ that allows its aromatic nuclei to be approximately coplanar and participate in $\pi-\pi$ stacking with the flavylium or quinonoidal forms of the anthocyanins (Liao et al., 1992). It has also been observed that procyanidin dimers with C4-C6 interflavanic linkages seem to be better copigments than their respective C4-C8 dimers (Berke \& de Freitas, 2005) and that galloylation at $\mathrm{C} 3$ of the catechin units improves the ability of flavan-3-ols to act as copigments (Berke \& De Freitas, 2005; Liao et al., 1992).

Different flavanols are preferentially extracted into the must during winemaking, depending on their location and solubility. It is known that different flavanol compositions exist in grape skins and seeds, the latter lacking gallocatechins and prodelphinidins and being richer in galloylated derivatives. On the other hand, flavanol extraction is easier from grape skin than from seeds, and galloylated derivatives are less soluble than the corresponding non-galloylated ones (Gonzalez-Manzano, Rivas-Gonzalo, \& Santos-Buelga, 2004). Thus, the levels and type of flavanols present in red wines will be highly dependent on the grape (type, maturity, etc.) and winemaking practices and, therefore, different extents of the copigmentation processes and their subsequent effect on the wine colour should be expected.

Most of the studies about copigmentation have been carried out in model solutions containing individual flavanols and anthocyanins, which makes it difficult to extrapolate the results to red wines. In this work the efficiencies of different flavanol mixtures obtained from the seeds and the skins of two Vitis vinifera grape varieties (Tempranillo and Graciano) to act as copigments of anthocyanin mixtures obtained from the same grape varieties were evaluated in model wine systems. The aim of this study was to ascertain the influence of the flavanols on the colour of the anthocyanins in conditions closer to those existing in wines.

\section{Materials and methods}

\subsection{Compound extraction}

Anthocyanins and flavanols were obtained from grapes of Graciano and Tempranillo varieties (Vitis vinifera) kindly supplied by Roda S.A. winery (Haro, La Rioja, Spain) and collected in the harvest of 2005 .

Anthocyanins were extracted from the grape skins with methanol: $\mathrm{HCl} 1 \mathrm{~N}(95: 5, \mathrm{v} / \mathrm{v})$ and further purified by semi-preparative HPLC, as described elsewhere (Heredia, Rivas-Gonzalo, Vicario, \& Santos-Buelga, 1998). Two different anthocyanin mixtures were obtained from each of the grape varieties used, which were further freeze-dried and their composition characterised by HPLC-DADMS. In addition, malvidin 3-O-glucoside (Mv3g) was also isolated from the same grape sources to be used in some copigmentation assays and as a standard for anthocyanin quantification.

For the preparation of flavanols, skins and seeds of the grape were manually separated and extracted with methanol:water $(75: 25, v / v)$. The methanol extracts were then submitted to liquid-liquid extraction with ethyl acetate; the ethyl acetate phase was collected, added to water and concentrated under vacuum. The final aqueous extracts were loaded onto a Sephadex LH-20 col- umn $(170 \times 20 \mathrm{~mm})$, which was first washed with water, and then flavanols were eluted successively with ethanol and acetone; the elution was monitored at $280 \mathrm{~nm}$. The flavanol extracts were further purified by semi-preparative HPLC in a Waters 600 chromatograph using a Phenomenex Ultracarb ODS20, $5 \mu \mathrm{m}(250 \times 10 \mathrm{~mm})$ column. The solvents were (A) $5 \%$ acetic acid and (B) methanol. The gradient used was $0-10 \%$ B over $10 \mathrm{~min} ; 10-20 \%$ B over $20 \mathrm{~min}$; 20-50\% B over $20 \mathrm{~min}$; $50-75 \%$ B over $5 \mathrm{~min}$ and $75-100 \%$ B over $5 \mathrm{~min}$, at a flow rate of $3 \mathrm{ml} / \mathrm{min}$. Detection was carried out at $280 \mathrm{~nm}$. The fractions that contained the flavanols were mixed, concentrated under vacuum and freeze-dried. Four flavanol extracts were finally obtained, corresponding to the skins and the seeds of each of the two varieties used, whose composition was characterised by HPLC-DAD-MS. The same protocol was also applied to isolate epicatechin (EC), procyanidin dimer B2 (EC-4,8EC) and B2-3'-O-gallate (EC-4,8-EC-3'-O-gallate), which were further used in some copigmentation assays and as standards for flavanol quantification.

\subsection{Preparation of model solutions}

Solutions of the anthocyanin mixtures obtained from each of the two considered grape varieties were prepared in $12 \%$ ethanol containing $5 \mathrm{~g} / \mathrm{l}$ of tartaric acid buffered with $1 \mathrm{M} \mathrm{NaOH}$ to $\mathrm{pH}$ 3.6 and ionic strength adjusted to $0.2 \mathrm{M}$ by addition of sodium chloride. To each of the anthocyanin solutions, flavanol mixtures from the seed and the skin obtained from the same grape variety were added at four concentration levels (0, 100, 200 and 400 $\mathrm{mg} / \mathrm{l})$. The final anthocyanin concentration was the same in all cases $(200 \mathrm{mg} / \mathrm{l})$. The solutions were transferred to a glass vial that was closed and stored in darkness at $25^{\circ} \mathrm{C}$. All solutions were prepared in triplicate. Spectrophotometric measurements were performed at $2 \mathrm{~h}$ and $1,2,3,4,6$ and 8 weeks of storage. Periodically the composition of the solutions was also checked by HPLC-DAD. The nomenclature used for the different solutions in Tables 2 and 3 was as follows: "AT" and "AG" refer to the solutions containing anthocyanin and flavanols obtained from Tempranillo and Graciano grapes, respectively; they are followed by a number that corresponds to the flavanol concentration in $\mathrm{mg} / \mathrm{l}(100,200$ or 400) accompanied by the letters "sd" or "sk" in accordance with their origin, either grape seed or skin, respectively.

\subsection{Copigmentation and colour analyses}

Spectral measurements were recorded in a Hewlett Packard UV-vis HP845x spectrophotometer (Palo Alto, CA, USA) using $2 \mathrm{~mm}$ path length glass cells thermostatted at $25^{\circ} \mathrm{C}$. Spectra were recorded in the whole visible range (380-770 nm) with $\Delta \lambda=1 \mathrm{~nm}$. The magnitude of the copigmentation was estimated by comparison of the absorbance at $520 \mathrm{~nm}$ from the anthocyanin solution $\left(A_{0}\right)$ and the absorbance at $520 \mathrm{~nm}$ from the solutions containing the different flavanol concentrations $(A)$ and expressed as the percentage:

$$
\left[\left(A-A_{0}\right) / A_{0}\right] \times 100
$$

The copigmentation effect was also evaluated by comparison of the absorbance at $520 \mathrm{~nm}$ in the different solutions before $(A)$ and after diluting them ten times with wine-like solution $(\mathrm{pH} 3.6)$ and multiplying by the dilution factor $\left(A_{\mathrm{d}}\right)$, also expressing the result as a percentage:

$$
\left[\left(A-A_{d}\right) / A_{d}\right] \times 100
$$

(Boulton, 1996). Colour was evaluated by calculation of the CIELAB parameters $\left(L^{*}, a^{*}, b^{*}, C_{a b}^{*}, h_{\mathrm{ab}}\right)$ from the visible spectra of the solutions, using the original CromaLab software (Heredia, 
Álvarez, González-Miret, \& Ramírez, 2004). The illuminant D65 and observed 10 were taken as references. Colour differences were also calculated as:

$\Delta E_{a b}^{*}=\left[\left(\Delta L^{*}\right)^{2}+\left(\Delta a^{*}\right)^{2}+\left(\Delta b^{*}\right)^{2}\right]^{1 / 2}$

\subsection{Thermodynamic measurements}

In order to determine the apparent hydration constant, model solutions of Mv3 g, previously isolated from grape skins, at a concentration of $1 \times 10^{-4} \mathrm{M}$, were prepared at different $\mathrm{pH}$ values (range 0.9-4.2) in citrate-phosphate buffer and the ionic strength adjusted to $1 \mathrm{M}$ with $\mathrm{NaCl}$. The solutions were kept for $2 \mathrm{~h}$ at $25^{\circ} \mathrm{C}$ to ensure complete hydration equilibrium and then their UV-Vis spectra were recorded as previously described. The value of first-order apparent rate constant of the hydration reaction $(K)$ is the sum of the hydration $\left(K_{\mathrm{h}}\right)$ and proton transference $\left(K_{\mathrm{a}}\right)$ constants $\left(K=K_{\mathrm{h}}+K_{\mathrm{a}}\right)$, and was deduced from plotting $A_{0} /\left(A_{0}-A\right)$ versus $10^{-\mathrm{pH}}$, resulting in a straight line, from the intercept to slope ratio, in accordance with Eq. (1), as described by Dangles, Saito, and Brouillard (1993).

$A_{0} /\left(A_{0}-A\right)=\left(K_{h}+K_{a}\left(1-r_{a}\right)\right)+\left(10^{-p H}\right) /\left(K_{h}+K_{a}\left(1-r_{a}\right)\right)$

In this equation, $A_{0}$ is the absorbance of a strongly acidic solution $(\mathrm{pH} \leqslant 1)$, in which the anthocyanin is in pure flavylium form and $A$ is the absorbance at a given slightly acidic $\mathrm{pH} ; r_{\mathrm{a}}$ is the ratio of molar absorption coefficients of the same pigment in its quinonoidal form and in its flavylium form.

Solutions of Mv3g $\left(1 \times 10^{-4} \mathrm{M}\right)$ were also prepared in citratephosphate buffer at $\mathrm{pH}$ values of 3.6 and 1 (ionic strength adjusted to $1 \mathrm{M}$ with $\mathrm{NaCl}$ ) containing increasing concentrations of different flavanols: EC, procyanidin dimer B2 and B2-3'-O-gallate, to obtain molar ratios flavanol:anthocyanin from $0: 1$ to $10: 1$. The solutions were kept for $2 \mathrm{~h}$ at $25^{\circ} \mathrm{C}$ to ensure complete hydration equilibrium and then their UV-Vis spectra were recorded. The values of the apparent $K$ of the Mv3g copigmented with different flavanols were calculated by substituting the $A$ values (absorbance at $\mathrm{pH}$ 3.6 of the solutions with different molar ratios of copigment: pigment from 0:1 to10:1) in Eq. (1). All assays were made in triplicate.

\subsection{HPLC-DAD-MS analyses}

Anthocyanin analysis was carried out using a Hewlett Packard 1100 chromatographic system with HP ChemStation. The column was a Waters Spherisorb C-18 ODS2, $3 \mu \mathrm{m}(4.6 \times 150 \mathrm{~mm})$. The solvents were (A) $0.5 \%$ trifluoroacetic acid (TFA) in water $(v / v)$ and (B) acetonitrile. The following linear gradient was used: $10 \%-15 \%$ B over $5 \mathrm{~min}, 15 \%$ B for $5 \mathrm{~min}, 15 \%$ to $18 \%$ B over $5 \mathrm{~min}$, $18 \%$ to $35 \%$ B over $25 \mathrm{~min}$ and $35 \%$ to $50 \%$ B over $10 \mathrm{~min}$, at $0.5 \mathrm{ml} / \mathrm{min}$ flow rate, followed by washing and reconditioning of the column. Detection was performed with a diode array detector, using $280 \mathrm{~nm}$ and $520 \mathrm{~nm}$ as preferred wavelengths. Quantification of the anthocyanins in the grape extracts obtained was made by comparison of the areas of their peaks recorded at $520 \mathrm{~nm}$ with an external calibration curve prepared with malvidin 3-O-glucoside previously isolated in the laboratory.

Flavanol analysis was carried out with the same chromatograph system and column thermostatted at $30^{\circ} \mathrm{C}$. The solvents were $(\mathrm{A})$ $2.5 \%$ acetic acid, (B) acetic acid/acetonitrile (10:90, v/v) and (C) acetonitrile. The flow rate was $0.5 \mathrm{ml} / \mathrm{min}$. The elution gradient established was $100 \%$ A to $100 \%$ B over $5 \mathrm{~min}, 0 \%-15 \%$ C in B over $25 \mathrm{~min}$, and $15 \%-50 \% \mathrm{C}$ in B over $10 \mathrm{~min}$. Detection was carried out at $280 \mathrm{~nm}$. Flavanols present in the extracts obtained from the skins and seeds of the grapes considered were quantified by comparison of the areas of their peaks recorded at $280 \mathrm{~nm}$ with external calibration curves prepared with different flavanol standards. Thus, catechin (C), epicatechin (EC) and non-galloylated procyanidin dimers (B1-B8) were quantified separately, using standards of each of them previously isolated in the laboratory, whereas gallocatechins and prodelphinidins, procyanidin trimers and galloylated derivatives were quantified by comparing the areas of their individual peaks (identified according to their UV and mass spectra) with calibration curves prepared with gallocatechin, trimer C1 and and B2-3'-O-gallate, respectively, which were further summed, to obtain the content of total compounds for every group.

\subsubsection{MS analyses}

The mass spectrometer was a Finnigan LCQ (Thermoquest, San Jose, CA) equipped with an ESI source and an ion-trap mass analyser, which were controlled by LCQ Navigator software. The system was connected to the probe of the mass spectrometer via the UV cell outlet. Both the auxiliary and the sheath gas were nitrogen at flow rates of 6 and $1.2 \mathrm{l} / \mathrm{min}$, respectively. The capillary voltage was $4 \mathrm{kV}$ and the capillary temperature $195^{\circ} \mathrm{C}$. Spectra were recorded in positive ion mode between $\mathrm{m} / \mathrm{z} 150$ and 2000. The MS detector was programmed to perform a series of three consecutive scans: an MS-MS scan of the most abundant ion in the first scan and an $\mathrm{MS}^{3}$ of the most abundant ion in the MS-MS, using a normalised collision energy of $45 \%$.

\subsection{Statistical analysis}

Analysis of variance was carried out using SPSS 13.0. Significant $(p<0.05)$ differences between means of three measurements were identified using the Tukey procedure.

\section{Results and discussion}

\subsection{Anthocyanin and flavanol composition in the prepared grape extracts}

The distribution of anthocyanins in the extracts obtained from Graciano and Tempranillo grape skins is shown in Table 1. Different anthocyanin profiles exist in each extract, which mostly reflect the composition of the original grape variety (Nunez, Monagas, Gomez-Cordoves, \& Bartolome, 2004). Thus, Graciano shows greater percentages of peonidin and malvidin 3-glucosides and hardly contains acetyl derivatives, whereas Tempranillo is richer in delphinidin 3-glucoside and acylated anthocyanins.

In the case of flavanols, catechin monomers ( $C$ and EC) and procyanidin dimers were quantified as individual compounds, whereas the groups of gallocatechins and prodelphinidins (PD), procyanidin trimers (TRIM) and galloylated derivatives (GAL) were considered jointly. Gallocatechins and prodelphinidins were identified in the chromatograms by their UV spectrum ( $\lambda_{\max }$ around 270-274 nm) and molecular ion. Among PD, mixed dimers from

Table 1

Distribution of anthocyanins (\%) in the extracts prepared from the skins of grapes of Graciano and Tempranillo varieties.

\begin{tabular}{lll}
\hline & Graciano & Tempranillo \\
\hline Delphinidin-3-glucoside & 15 & 27 \\
Cyanidin-3-glucoside & 2.8 & 4.4 \\
Petunidin-3-glucoside & 13.6 & 15.2 \\
Peonidin-3-glucoside & 15.6 & 5.1 \\
Malvidin-3-glucoside & 44.3 & 34.2 \\
Delphinidin-3-acetylglucoside & & 2 \\
Malvidin-3-acetylglucoside & & 1.4 \\
Delphinidin-3-coumaroylglucoside & & 2.3 \\
Peonidin-3-coumaroylglucoside & 5.5 & \\
Malvidin-3-coumaroylglucoside & 3.2 & 9.4 \\
\hline
\end{tabular}


(epi)catechin and (epi)gallocatechin (molecular ion at $\mathrm{m} / \mathrm{z} 595$ ) were the predominant compounds in the skin extracts, although smaller levels of monomers ( $m / z$ at 307 ), (epi)gallocatechin dimers $(\mathrm{m} / \mathrm{z}$ at 611$)$ and trimers $(\mathrm{m} / \mathrm{z}$ at 883$)$ were also present. Among the procyanidin trimers ( $\mathrm{m} / \mathrm{z}$ at 867 ), the presence of EC-4,8-EC-4,8-EC (C1) and EC-4,8-EC-4,8-C was predominant, accompanied by minor amounts of other non-characterised compounds. Galloylated compounds were mostly represented by EC-3-O-gallate, B1-3-O-gallate (predominant in the skins) and B2-3'-O-gallate (predominant in the seeds).

The distribution of flavanols in the extracts prepared from the skins and seeds of each of the grape varieties are presented in the Fig. 1 . The extracts from the skin of the Graciano variety were characterised by the presence of greater amounts of EC than C and higher percentages of gallocatechins and prodelphinidins and procyanidin trimers and lower proportions of galloylated derivatives compared to the extracts from Tempranillo. Regarding the extracts prepared from the grape seeds (Fig. 1b), the one obtained from Graciano contained lower proportions of catechin monomers and greater percentages of procyanidin dimers and trimers and galloylated derivatives than Tempranillo. These profiles roughly reflect the distribution of flavanols of low degree of polymerisation in the original grapes.

\subsection{Copigmentation between anthocyanins and flavanols from grape} skins and seeds

The addition of the flavanol mixtures, from either grape seed or skin, to the solutions of anthocyanins from Graciano and Tempranillo grapes produced a hyperchromic shift in the visible region of the spectrum, more pronounced as the concentration of flavanols increased in the solutions, indicating the existence of a copigmentation process. Fig. 2 shows the magnitude of this effect observed $2 \mathrm{~h}$ after preparation of the solutions and expressed as $\left[A-A_{0} / A_{0}\right] \times 100$.
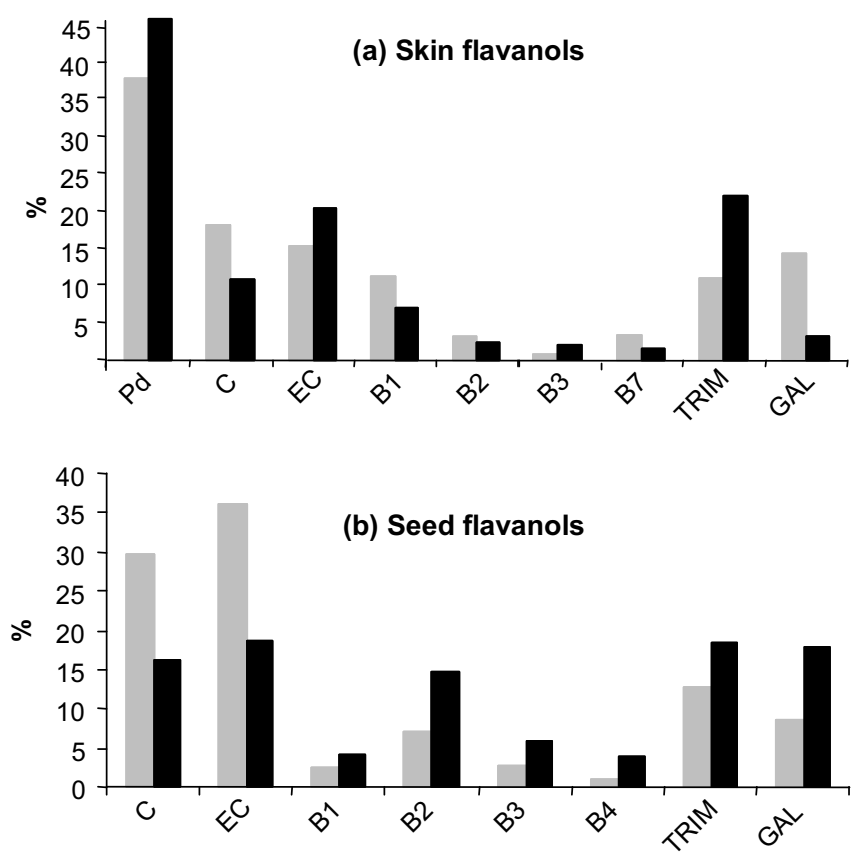

Fig. 1. Distribution of flavanols in the extracts prepared from the skins (a) and seeds (b) of grapes of the varieties Graciano (grey bars) and Tempranillo (black bars). Abbreviations: PD, gallocatechins + prodelphinidins; C: catechin; EC: epicatechin; B1, B2, B3, B7: procyanidin dimers; TRIM: procyanidin trimers; GAL: galloyled derivatives.
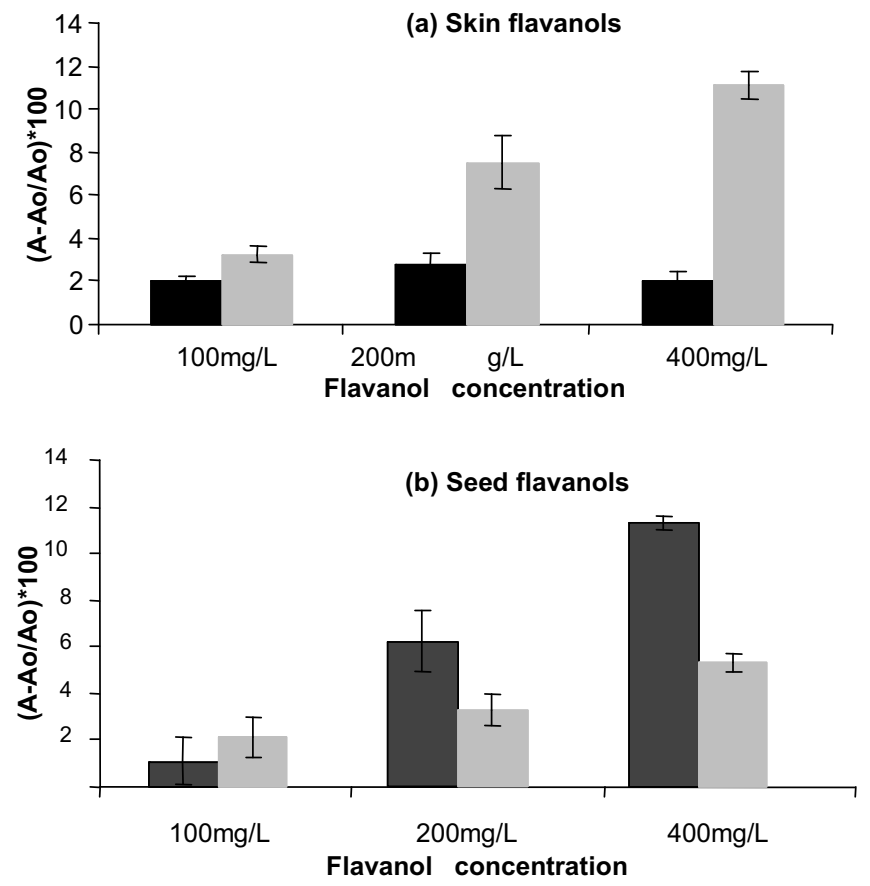

Fig. 2. Magnitude of the copigmentation effect between anthocyanins and flavanols obtained from the skins (a) and seeds (b) of grapes of the varieties Graciano (grey bars) and Tempranillo (black bars), evaluated as the ratio $\left[\left(A-A_{0}\right) / A_{0}\right] \times 100,2 \mathrm{~h}$ after preparation of the solutions.

In the case of flavanols obtained from grape skins a greater copigmentation effect was observed for the extracts from Graciano than for those of Tempranillo, whereas the opposite was found for the extracts prepared from the seeds, where Tempranillo was more effective. This observation confirms that not only the concentrations of flavanols but also their qualitative profiles have a strong influence on the copigmentation effect. In each case the most efficient extracts were richer in flavanols suggested as better anthocyanin copigments, like epicatechin (Brouillard et al., 1991; Liao et al., 1992; Mirabel et al., 1999) or galloylated procyanidins (Berke \& De Freitas, 2005; Liao et al., 1992; Mistry et al., 1991).

Skin flavanols are known to be more available than seed flavanols, whereas galloylated derivatives are more difficult to extract than the equivalent non-galloylated ones (Gonzalez-Manzano et al., 2004). This would suggest that the wines from Tempranillo grapes would benefit from longer maceration times than those from Graciano to obtain a greater copigmentation effect, as provided by the flavanols of their seeds. This suggestion is coherent with observations made in red wines obtained from both grape varieties using variable maceration times (Gonzalez-Manzano, Santos-Buelga, Pérez-Alonso, Rivas-Gonzalo, \& Escribano-Bailón, 2006).

The behaviour of the solutions was followed over 8 weeks (Fig. 3), showing that maximum hyperchromic effect was not obtained immediately after preparation of the solutions but around 1 -weeks, except in the case of the solutions copigmented with flavanols from the seeds of Tempranillo, in which the maximum effect was observed at $4-8$ weeks (Fig. $3 b$ ). The observation that the copigmentation is not an immediate phenomenon, but it is progressively established, was already observed in previous studies by our group (González-Manzano, Santos-Buelga, Dueñas, RivasGonzalo, \& Escribano-Bailón, 2008; Gómez-Míguez et al., 2006) and points out that the process was controlled thermodynamically rather than kinetically.

The existence of a copigmentation process was not the cause of a decrease in the anthocyanins produced in the solutions. Similar 

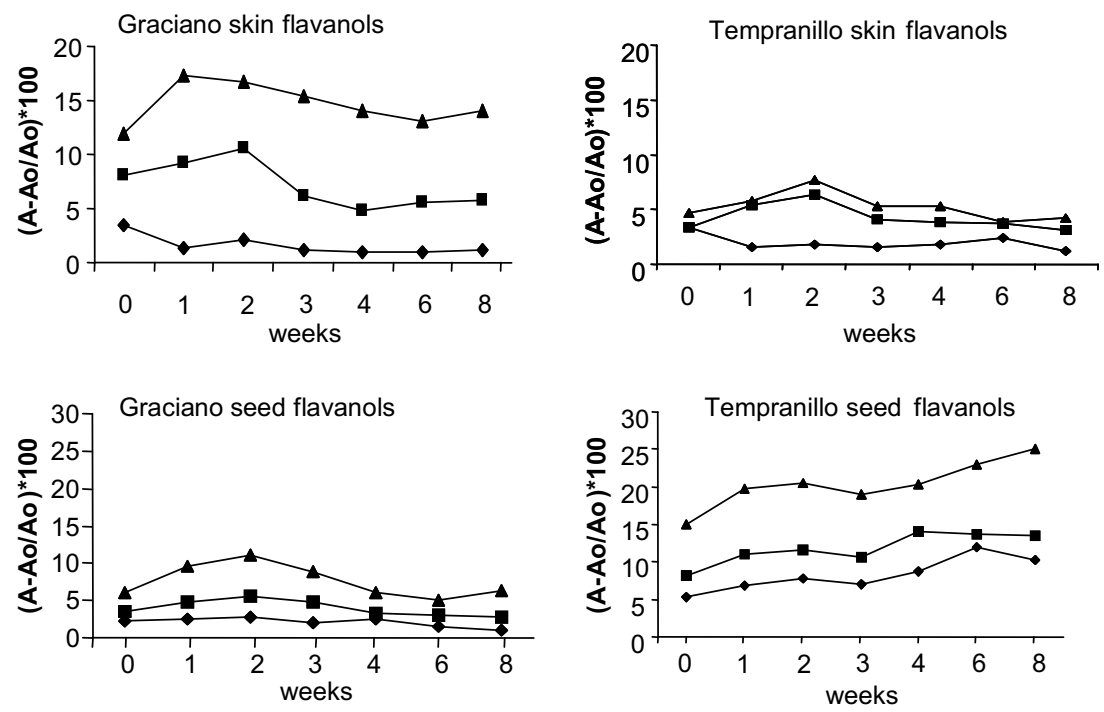

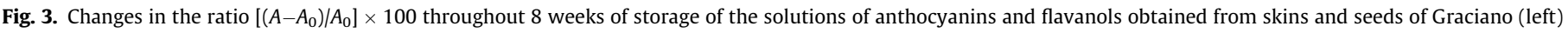
and Tempranillo (right).

behaviour of the anthocyanins was found, independent of the presence of flavanols in the medium. A final decrease in the anthocyanin content of $55 \%$, on average, was produced after 8 weeks of assay, irrespective of the copigments present. Also, no significant differences in the anthocyanin contents were found at any of the assayed time points among the different solutions. Similar observations had already been made in previous studies (Gómez-Míguez et al., 2006). Other authors found that the addition of rutin to red wines during winemaking had a stabilising effect on the anthocyanins contents (Schwarz, Picazo-Bacete, Winterhalter, \& HermosinGutierrez, 2005). Thus, the usual assumption that the formation of copigmentation complexes is a protective factor against anthocyanin disappearance in wines may not be true in all cases and depend on the type of copigments and the strength of the interaction established. The fact that the magnitude of the copigmentation (evaluated by the ratio $\left[\left(A-A_{0}\right) / A_{0}\right]$ ) does not decline in our studies with the observed decrease in the concentration of anthocyanins, but can even increase, would support the previous indication about the progressive formation of copigmentation complexes between anthocyanins and flavanols.

An additional factor that might contribute to the maintenance of the absorbance in the solutions is the formation of new products. The presence in the chromatograms of some minor new peaks that absorb in the visible range began to be observed in the model solutions after the third week of storage. Thus, compounds whose spectral and mass characteristics coincided with the vinylpyranoanthocyanins of Mv3g $(\mathrm{m} / z$ at 517$)$ and its $p$-coumarate $(\mathrm{m} / z$ at $663)$, and Mv3g-ethyl-(epi)catechin adducts ( $\mathrm{m} / \mathrm{z}$ at 809 ) were detected in all the solutions, while the vinylpyranoanthocyanins of peonidin 3-glucoside $(\mathrm{m} / \mathrm{z}$ at 487$)$ and its $p$-coumarate $(\mathrm{m} / \mathrm{z}$ at 632 ) were only observed in the solutions prepared with anthocyanins from Graciano grapes. The concentrations of all these new pigments in the solutions were very low and could not be quantified precisely. Nevertheless, their greater resistance to the bleaching effect of $\mathrm{pH}$ than the original anthocyanins might make them able to contribute in some extent to the colour of the solutions.

\subsection{Copigmentation and self-association}

In a previous paper, we showed that self-association explained a part of the colour expression of anthocyanins in wine-like media at concentrations similar to those occurring in red wines (Gon-
zález-Manzano et al., 2008). The self-association is demonstrated as a positive deviation from Beeŕs law and it can be evaluated through the difference of absorbance at the anthocyanin maximum wavelength $\left(\lambda_{\max }\right)$ in the visible range of the solutions before $(A)$ and after being diluted and later multiplied by the dilution factor $\left(A_{\mathrm{d}}\right)$, and expressed as the ratio $\left[\left(A-A_{\mathrm{d}}\right) / A_{\mathrm{d}}\right] \times 100$. The dilution "breaks" all the anthocyanin complexes, either intermolecular or among anthocyanins themselves. Therefore, in media where different copigments exist, the relation $\left[\left(A-A_{\mathrm{d}}\right) / A_{\mathrm{d}}\right] \times 100$ should jointly evaluate the effect of copigmentation and self-association processes, whereas the ratio $\left[A-A_{0}\right] / A_{0} \times 100$ would only evaluate intermolecular copigmentation. With the aim of verifying this assumption, the ratio $\left[\left(A-A_{\mathrm{d}}\right) / A_{\mathrm{d}}\right] \times 100$ was also calculated in the different solutions prepared and compared with the values obtained for the ratio $\left[A-A_{0}\right] / A_{0} \times 100$ after the preparation of the solutions and after 2 weeks of storage, at which the maximum hyperchromic effect at $\lambda_{\max }$ in the visible range was reached in most solutions. The results are shown in the Table 2 . It can be seen that the difference between the values of the ratio $\left[\left(A-A_{\mathrm{d}}\right) /\right.$ $\left.A_{\mathrm{d}}\right] \times 100$ obtained in the presence of flavanols (i.e., copigmentation + self-association) and in their absence (i.e., self-association) are in good agreement with those obtained for the ratio $\left[A-A_{0}\right] /$ $A_{0} \times 100$ (i.e., copigmentation). These similar results show the adequacy of both approaches for the estimation of the copigmentation process.

The values of both ratios increased with the time of storage, supporting the view that both copigmentation and self-association were not immediately established. On the other hand, the deviation between the values of the copigmentation obtained with each way of calculation was higher in the measurements made after two weeks, at which the values of the relation $\left[A-A_{0}\right] / A_{0}$ were slightly higher than those obtained from the calculation performed for dilutions of the solutions. This may be interpreted as there could be some competition between self-association and copigmentation and a part of the self-association complexes was displaced by the intermolecular interactions.

The results obtained show that a relevant part of the colour expression of the anthocyanins in wine-like solutions can be explained by self-association processes, whose influence on the colour is expected to increase with the increase of the anthocyanins concentration in the medium. Another observation made is that for a similar concentration of anthocyanins the self-association 
Table 2

Evaluation of the magnitudes of copigmentation and self-association in the different solutions of anthocyanins from Graciano (AG) and Tempranillo (AT) grapes obtained at $2 \mathrm{~h}$ and 2 weeks after the preparation of the solutions. Tables on the left side show the results obtained for the ratio $\left(A-A_{d} \mid A_{d}\right) \times 100$ in the absence (self-association) and presence of different concentrations of flavanols obtained from the skins and seeds of the same grape varieties (self-association + copigmentation); the difference between both values is attributed to intermolecular copigmentation (Copig). Tables on the right side show the results obtained for the ratio $\left(A-A_{0} / A_{0}\right) \times 100$ in the solutions containing anthocyanins and flavanols and are only associated with copigmentation. For nomenclature of the solutions see the Section 2.

\begin{tabular}{|c|c|c|c|c|c|}
\hline $2 \mathrm{~h}$ & & $\left(A-A_{d} / A_{d}\right) \times 100$ & Copig & $2 \mathrm{~h}$ & $\left(A-A_{0} / A_{0}\right) \times 100$ \\
\hline Grac & AG & 9 & & Grac & \\
\hline & AG100sd & 11 & 2 & AG100sd & 2 \\
\hline & AG200sd & 12 & 3 & AG200sd & 4 \\
\hline & AG400sd & 14 & 5 & AG400sd & 6 \\
\hline & AG100sk & 12 & 3 & AG100sk & 3 \\
\hline & AG200sk & 16 & 7 & AG200sk & 8 \\
\hline & AG400sk & 19 & 10 & AG400sk & 12 \\
\hline Temp & AT & 6 & & Temp & \\
\hline & AT100sd & 7 & 1 & AT100sd & 1 \\
\hline & AT200sd & 12 & 6 & AT200sd & 7 \\
\hline & AT400sd & 18 & 12 & AT400sd & 13 \\
\hline & AT100sk & 7 & 1 & AT100sk & 2 \\
\hline & AT200sk & 9 & 3 & AT200sk & 3 \\
\hline & AT400sk & 9 & 3 & AT400sk & 4 \\
\hline 2 weeks & & $\left(A-A_{d} / A_{d}\right) \times 100$ & Copig & 2 weeks & $\left(A-A_{0} / A 0\right) \times 100$ \\
\hline Grac & AG & 27 & 0 & Grac & \\
\hline & AG100sd & 27 & 0 & AG100sd & 3 \\
\hline & AG200sd & 29 & 2 & AG200sd & 6 \\
\hline & AG400sd & 36 & 9 & AG400sd & 11 \\
\hline & AG100sk & 27 & 0 & AG100sk & 2 \\
\hline & AG200sk & 36 & 9 & AG200sk & 10 \\
\hline & AG400sk & 41 & 14 & AG400sk & 17 \\
\hline Temp & AT & 18 & 0 & Temp & \\
\hline & AT100sd & 24 & 6 & AT100sd & 8 \\
\hline & AT200sd & 28 & 10 & AT200sd & 12 \\
\hline & AT400sd & 34 & 16 & AT400sd & 20 \\
\hline & AT100sk & 18 & 0 & AT100sk & 2 \\
\hline & AT200sk & 19 & 1 & AT200sk & 6 \\
\hline & AT400sk & 21 & 3 & AT400sk & 8 \\
\hline
\end{tabular}

effect is higher in the mixtures of anthocyanins obtained from the Graciano variety than from Tempranillo. This confirms again that the qualitative composition of anthocyanins also plays an important role in the colour definition, as previously suggested in the studies carried out with individual anthocyanins (González-Manzano et al., 2008).

\subsection{Influence of copigmentation on the colour}

With the aim of evaluating the actual effect of the copigmentation processes on the colour, chromatic analyses in the CIELAB space were performed. Table 3 collects the values of the colorimetric parameters, $L^{*}, C_{a b}^{*}, h_{\mathrm{ab}}$ and $\Delta E_{a b}^{*}$, for the different solutions calculated after their preparation $(2 \mathrm{~h})$ and at $1,2,4$ and 8 weeks of storage. The copigmentation produced a decrease in the lightness $L^{*}$ (darkening) and an increase in the values of chroma $C_{a b}^{*}$ (greater amount of colour). The hyperchromic shift in the spectra of the solutions was not accompanied by important modifications in their shape and, for that reason, the changes produced in the hue values, $h_{\mathrm{ab}}$, between the anthocyanin solutions in the absence and presence of flavanols were not very important, although, with the passage of time, a certain displacement towards orange tonalities was observed. In any case, under the assay conditions used, the colour changes induced were more quantitative $\left(\Delta L^{*}\right.$ and $\left.C_{a b}^{*}\right)$ than qualitative $\left(h_{\mathrm{ab}}\right)$. Similar effects of the copigmentation process on the CIELAB parameters were also observed by other authors (GómezMíguez et al., 2006; Berke \& De Freitas, 2005; Gonnet, 1998). All
Table 3

Colorimetric parameters $L^{*}, C_{a b}^{*}, h_{a b}$ and $\Delta E_{a b}^{*}$ obtained in the solutions prepared with anthocyanins and flavanols obtained from Graciano (left table) and Tempranillo (right table) grape varieties at $2 \mathrm{~h}, 1,2,4$ and 8 weeks of assay. Nomenclature of the solutions as in Table 2.

\begin{tabular}{|c|c|c|c|c|c|c|c|c|c|}
\hline $2 \mathrm{~h}$ & $L^{*}$ & $C_{a b}^{*}$ & $h_{a b}$ & $\Delta E_{a b}^{*}$ & $2 \mathrm{~h}$ & $L^{*}$ & $C_{a b}^{*}$ & $h_{a b}$ & $\Delta E_{a b}^{*}$ \\
\hline G & 2.7 & 45.6 & 15.2 & & AT & 36.1 & 27.3 & 4.4 & \\
\hline AG100sk & 49.6 & 46.1 & 15.2 & 7.4 & AT100sk & 34.6 & 27.3 & 5.2 & 1.6 \\
\hline G200sk & 46.4 & 47.5 & 15.9 & 12.1 & AT200sk & 34.4 & 27.6 & 5.5 & 1.9 \\
\hline G400sk & 45.8 & 49.6 & 19 & 14.6 & AT400sk & 34.9 & 28 & 5.5 & 1.5 \\
\hline AG100sd & 49.1 & 45.7 & 15.6 & 8.6 & AT100sd & 34.8 & 27.5 & 5.2 & 2.3 \\
\hline AG200sd & 48.9 & 45.2 & 14.5 & 10 & AT200sd & 31.8 & 29.1 & 5.5 & 5.8 \\
\hline AG400sd & 46.9 & 45.2 & 17.2 & 13.5 & AT400sd & 30.1 & 30.1 & 6.5 & 9.1 \\
\hline \multicolumn{5}{|l|}{1 week } & \multicolumn{5}{|l|}{1 week } \\
\hline G & 45.2 & 44 & 27.1 & & AT & 36.4 & 23.3 & 18.2 & \\
\hline G100sk & 44.8 & 46.5 & 27.9 & 3.9 & AT100sk & 35.8 & 23.6 & 18.4 & 0.6 \\
\hline G200sk & 42.2 & 48.6 & 29.6 & 7.6 & AT200sk & 33.7 & 24 & 18.8 & 2.8 \\
\hline G400sk & 38.4 & 51.2 & 30 & 13.5 & AT400sk & 33.5 & 24.1 & 19.9 & 3.1 \\
\hline G100sd & 44.1 & 45 & 28.4 & 3 & AT100sd & 33.8 & 26.5 & 20.4 & 3.6 \\
\hline AG200sd & 43.4 & 46.7 & 28.2 & 5.9 & Osd & 31.3 & 8.6 & & 7.2 \\
\hline AG400sd & 41.8 & 50.1 & 28.1 & 10.8 & AT400sd & 27.6 & 29.2 & 22.5 & 12 \\
\hline \multicolumn{5}{|l|}{2 weeks } & \multicolumn{5}{|l|}{2 weeks } \\
\hline AG & 3.9 & 45.3 & 36 & & & & 2.3 & & \\
\hline G100sk & 44.3 & 48.6 & 36.1 & 4.8 & AT100sk & 0.5 & 23.9 & 15.3 & 2.3 \\
\hline G200sk & 38.7 & 49.2 & 39.1 & 8.8 & AT200sk & 38.2 & 23.6 & 16 & 2.3 \\
\hline AG400sk & 37.9 & 53.3 & 39.7 & 13.6 & AT400sk & 37.8 & 23.8 & 18.5 & 2.5 \\
\hline AG100sd & 43.2 & 47.5 & 36.4 & 4.3 & Osd & 36 & 24.3 & 20.8 & 4.5 \\
\hline AG200sd & 41.5 & 48.9 & 38.6 & 7 & AT200sd & 34.1 & 25.5 & 21.2 & 8.2 \\
\hline AG400sd & 40.1 & 52.8 & 39.6 & 12.4 & AT400sd & 29.9 & 26.8 & 21.4 & 12.9 \\
\hline \multicolumn{5}{|l|}{4 weeks } & \multicolumn{5}{|l|}{4 weeks } \\
\hline 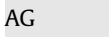 & 9.5 & 44.2 & 35.3 & & & 5.2 & 20.8 & 26.2 & \\
\hline Osk & 42.6 & 49.1 & 40.9 & 7. & sk & & 21.8 & 25.5 & 1 \\
\hline AG200sk & 38.5 & 50.4 & 43 & 8.9 & Osk & 34.3 & 22.3 & 26.3 & 1.7 \\
\hline AG400sk & 35.8 & 54.6 & 45.1 & 15 & AT400sk & 33.5 & 22.7 & 28.9 & 2.7 \\
\hline AG100sd & 41.5 & 48.8 & 37.1 & 7.8 & AT100sd & 32.1 & 22.8 & 27.1 & 4.3 \\
\hline AG200sd & 41.2 & 51.6 & 40.8 & 11.4 & AT200sd & 29.6 & 25.1 & 28.4 & 8.3 \\
\hline AG400sd & 38.9 & 55.8 & 42.3 & 13.6 & AT400sd & 26 & 27 & 29.3 & 12.9 \\
\hline \multicolumn{5}{|l|}{8 weeks } & \multicolumn{5}{|l|}{8 weeks } \\
\hline AG & 9.4 & 40.7 & 37.7 & & . & - & 19.1 & 35.5 & \\
\hline AG100sk & 39.1 & 46.4 & 39.7 & 8 & AT100sk & 33.7 & 20 & 37.5 & 1.3 \\
\hline AG200sk & 34.2 & 47.3 & 45.8 & 10.1 & AT200sk & 32.9 & 21.1 & 38.4 & 2.2 \\
\hline AG400sk & 31.5 & 51.8 & 47.6 & 16.3 & AT400sk & 33 & 22.5 & 39.2 & 4 \\
\hline AG100sd & 39.7 & 48.3 & 38.9 & 10.2 & AT100sd & 30.3 & 22.2 & 38.3 & 4.7 \\
\hline AG200sd & 39.8 & 51.7 & 40.9 & 14.5 & AT200sd & 29.5 & 25.9 & 39.9 & 8.8 \\
\hline AG400sd & 36.9 & 56.4 & 43.3 & 19.9 & AT400sd & 23.2 & 27.6 & 40.4 & 14.3 \\
\hline
\end{tabular}

these changes were more pronounced with the increase of the concentration of flavanols in the medium and the time of storage, which was coherent with the results obtained about magnitude of the copigmentation when evaluated by the effect at maximum wavelength of absorption.

In order to establish whether the observed changes in the chromatic parameters were visually relevant, the colour differences, $\Delta E_{a b}^{*}$, between solutions containing only anthocyanins and those that also had flavanols, were calculated. It has been indicated that a non-trained human eye is able to distinguish two colours with $\Delta E_{a b}^{*}$ values of 3.0 units CIELAB (Martínez, Melgosa, Pérez, Hita, \& Negueruela, 2001). Thus, the results shown in Table 3 suggest that the colour changes induced by the presence of flavanols in the anthocyanin solutions were perceptible in all the preparations made with compounds obtained from the Graciano at all the assayed concentrations, as well as in most of the solutions containing Tempranillo seed flavanols. By contrast, most of the solutions containing flavanol from Tempranillo skins did not surpass the value of 3.0 units CIELAB for $\Delta E_{a b}^{*}$, which confirms their poor efficiency as anthocyanin copigments.

Peculiarly, the increases in diverse chromatic parameters and $\Delta E_{a b}^{*}$ values produced by Tempranillo seed flavanols were less (or 
of same order) than those induced for Graciano seed flavanols. This does not agree with the results obtained when the copigmentation was evaluated from the hyperchromic shift at anthocyanin $\lambda_{\max }$ (relation $\left.\left[A-A_{0}\right] / A_{0}\right)$ discussed above. An explanation for this fact must be that the copigmentation measurement, as an evaluation of changes at a unique wavelength, does not show the integral colour behaviour, on which the changes produced in all the visible spectrum range have influence. This noteworthy observation once again confirms the importance of carrying out a suitable evaluation of the copigmentation effect and not being satisfied only with measurements at specific wavelengths, as usually employed, which can only give an indication of the phenomenon (Gonnet, 1998; GómezMíguez et al., 2006).

Another interesting observation was that when the copigmentation was evaluated from the changes at $\lambda_{\text {max }}$, from the third week of assay no increase in the copigmentation effect was observed when Graciano skin and seed flavanols were used. However, at this stage colour changes continued to be produced when the evaluation was made by colour measurements in the CIELAB space. There has been no definitive explanation for this behaviour, although the presence of new pigments in the solutions might account for it.

All in all, in relation to the colour effects it can be concluded that skin and seed Graciano flavanols have greater effectiveness as anthocyanin copigments than those of Tempranillo, since they induce more important and more easily perceptible colour changes. Once more this shows that not only the amount but also the qualitative flavanol composition has a determining influence on the colour of the anthocyanin solutions, stressing the importance of a suitable variety selection to obtain the results sought with relation to the colour. It should be borne in mind that concentrations of anthocyanins and flavanols used in these assays are in the range in which they can be found in wines, thus a possibility is that similar copigmentation effects and influences on the colour can be expected in them.

\subsection{Thermodynamic measures}

Copigmentation modifies the equilibria of distribution among the different structural forms of the anthocyanins in relation to the $\mathrm{pH}$, limiting the hydration of the flavylium cation, which results in the existence in the medium of a lower proportion of colourless hydrated forms than could be expected in accordance with the existing $\mathrm{pH}$. This equilibria displacement should be reflected in an increase in the $p K_{\mathrm{h}}$ of the anthocyanins. As a consequence, the acidity equilibrium of the flavylium cation should also be modified, so that the proportion of quinoidal forms in the medium increases. Therefore, the changes in the hydration $\left(K_{\mathrm{h}}\right.$, conversion of ion flavylium cation to carbinol pseudobase) and acidity $\left(K_{\mathrm{a}}\right.$, conversion of ion flavylium to quinoidal base) constants of the anthocyanins can also be employed as a measure of the copigmentation phenomenon. The predominance of the hydration reaction over the rest of the equilibria makes $K_{\mathrm{a}}$ very small compared with $K_{\mathrm{h}}\left(K_{\mathrm{a}}<<K_{\mathrm{h}}\right)$, so the two constants can be assimilated to the apparent equilibrium constant $\left(K_{\mathrm{h}}+K_{\mathrm{a}}\right)$.

In order to determine the modification of the anthocyanin equilibria in the presence of flavanols, assays were also carried out in solutions of Mv3g $\left(1 \times 10^{-4} \mathrm{M}\right.$ in citrate-phosphate buffer solution, $\mathrm{pH} 3.6$ and ionic strength adjusted to $1 \mathrm{M}$ by addition of sodium chloride) alone and with different added concentrations of EC, procyanidin B2 and B2-3'-O-gallate at molar relations flavanol:anthocyanin from $0: 1$ to 10:1. A low concentration of the anthocyanin was used in these assays to prevent self-association and thus evaluate the copigmentation effect without interference. Table 4 shows the anthocyanins' $\mathrm{p} K_{\mathrm{h}}$ values in the presence of the three assayed flavanols for the different molar relations employed. It is possible to verify that, in general, $\mathrm{p} K_{\mathrm{h}}$ increases when the proportion of copigment also increases in the solution, from a value of 2.93, corresponding to Mv3g alone, to a maximum value of 3.36 obtained in the solution with the maximum concentration of B23'-O-gallate. Significant differences between the three flavanols for equal ratios of copigment:pigment exist, as well as between the different ratios for the same copigment, with minor exceptions. It is thus possible to conclude that even compounds considered poor copigments, like flavanols, induce a significant displacement of the anthocyanin equilibria towards coloured forms. Among the compounds tested, B2-3'-O-gallate would behave as the best anthocyanin copigment, followed by EC and procyanidin B2. This result confirms previous observations of other authors regarding the relative efficiency of these compounds as anthocyanin copigments (De Freitas, Glories, \& Laguerre, 1998; Liao et al., 1992).

For a better visualisation of how the equilibria between anthocyanin forms are modified by the copigmentation process, in Fig. 4 the proportions $\left(\chi_{\mathrm{ah}}\right)$ of the anthocyanin coloured forms as a function of the type and concentration of copigment are presented. As an example, it can be seen that for a flavanol:anthocyanin molar ratio of $5: 1$, at $\mathrm{pH} 3.6$, the percentages of coloured forms of the anthocyanin in the medium constitute $31 \%$ in the presence of

\section{Table 4}

Values of the $p K_{\mathrm{h}}$ of Mv3g in the presence of increasing concentrations of three flavanol copigments (EC, B2 and B2-3'-O-gallate) in wine-like solutions of pH 3.6. Different letters in the columns (a-f) and different symbols in the rows $(\otimes, \oplus, \varnothing)$ indicate significant differences in the Tukey test $(p<0.05)$

\begin{tabular}{llll}
\hline $\mathrm{pK}_{\mathrm{h}}$ & & & \\
\hline Flavanols:Mv3g & EC & B2 & B2-3́o-gal \\
\hline $1: 0$ & $2.93 \pm 0.0036^{\mathrm{a} \otimes}$ & $2.93 \pm 0.0014^{\mathrm{a} \otimes}$ & $2.93 \pm 0.0014^{\mathrm{a} \otimes}$ \\
$1: 1$ & $2.97 \pm 0.0051^{\mathrm{b} \otimes}$ & $2.96 \pm 0.0012^{\mathrm{b} \oplus}$ & $3.01 \pm 0.0012^{\mathrm{b} \varnothing}$ \\
$2: 1$ & $2.97 \pm 0.0068^{\mathrm{b} \otimes}$ & $2.97 \pm 0.0015^{\mathrm{c} \otimes}$ & $3.07 \pm 0.0000^{\mathrm{c} \varnothing}$ \\
$5: 1$ & $3.06 \pm 0.0075^{\mathrm{c} \otimes}$ & $3.04 \pm 0.0001^{\mathrm{d} \oplus}$ & $3.19 \pm 0.0045^{\mathrm{d} \varnothing}$ \\
$7: 1$ & $3.11^{\mathrm{d}} \pm 0.0055^{\mathrm{d} \otimes}$ & $3.07 \pm 0.0006^{\mathrm{e} \oplus}$ & $3.27 \pm 0.0028^{\mathrm{e} \varnothing}$ \\
$10: 1$ & $3.20 \pm 0.0040^{\mathrm{e} \otimes}$ & $3.12 \pm 0.0006^{\mathrm{f} \oplus}$ & $3.36 \pm 0.0028^{\mathrm{f} \varnothing}$ \\
\hline
\end{tabular}
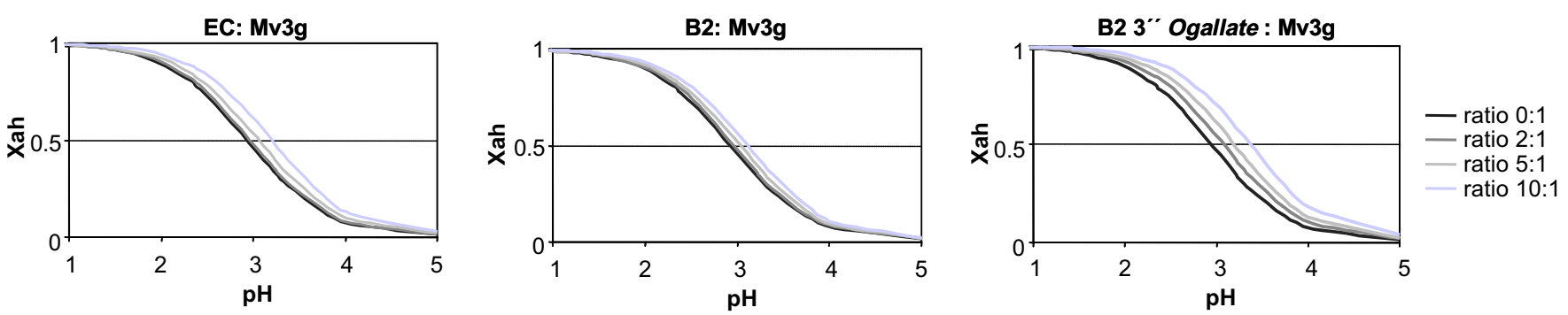

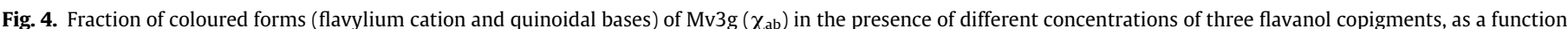

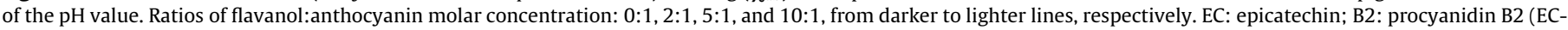
4,8-EC); B2-3-gal: EC-4,8-EC-3,0-gallate. 
B2-3-O-gallate, $25 \%$ with EC, and $24 \%$ with B2, compared with $20 \%$ in $\mathrm{Mv3g}$ solution when no flavanol is present.

\section{Conclusions}

The copigmentation effect between anthocyanins and flavanols is determined by the quantitative and qualitative composition of both types of compounds in the medium. In addition to the copigmentation phenomenon, for the anthocyanin colour expression, the contribution of the self-association process should also be considered. In general, it has been shown that both processes take place more favourably in the case of the Graciano phenolics than in those of Tempranillo. Regarding the colour, the copigmentation effect causes a decrease in lightness, $L^{*}$, increases in chroma $C_{a b}^{*}$ and, with the passage of time, a tone shift towards orange tonalities. In most of the solutions assayed, colour differences perceptible to the human eye were observed. It was shown that flavanols, considered poor anthocyanin copigments, can induce significant modification in the colour of the anthocyanin in winelike solutions at concentrations similar to those that can exist in red wines. It was also observed that the evaluation of the copigmentation effect made by chromatic analysis in CIELAB colour space showed some divergences from that carried out by means of classical approaches based on the measurement of absorbance at anthocyanin $\lambda_{\text {max }}$. This is because the latter approach does not consider the integral colour changes produced by the modification of the spectrum in the visible region range, which reaffirms the interest in making a suitable evaluation of the process to show the real colour modifications and their incidence on visual perception. The full set of observations made confirm the importance of the qualitative phenolic composition, determined in the wines by the type of grape and winemaking practices, to the production of an effective copigmentation process.

\section{Acknowledgements}

Financial support received from CICYT (Grant ref. AGL200507245-C03-01). Thanks are also due to Mr. G.H. Jenkins for his assistance with the English version of the manuscript.

\section{References}

Asen, S., Stewart, R. N., \& Norris, K. H. (1972). Co-pigmentation of anthocyanins in plant tissues and its effects on color. Phytochemistry, 11, 1139-1144.

Berke, B., \& De Freitas, V. A. P. (2005). Influence of procyanidin structures on their ability to complex with oenin. Food Chemistry, 90(3), 453-460.

Boulton, R. (2001). The copigmentation of anthocyanins and its role in the color of red wine: A critical review. American Journal of Enology and Viticulture, 52, 67-84.

Boulton, R. B. (1996). A method for the assessment of copigmentation in red wines. Presented at the Forty-seventh Annual Meeting of the American Society for Enology and viticulture, Reno, NV, 1966.
Brouillard, R., \& Dangles, O. (1994). Anthocyanin molecular-interactions - the first step in the formation of new pigments during wine aging. Food Chemistry, 51(4), 365-371.

Brouillard, R., Wigand, M. C., Dangles, O., \& Cheminat, A. (1991). pH and solvent effects on the copigmentation reaction of malvin with polyphenols, purine and pyrimidine-derivatives. Journal of the Chemical Society-Perkin Transactions, 2(8), 1235-1241.

Dangles, O., Saito, N., \& Brouillard, R. (1993). Kinetic and thermodynamic control of flavylium hydration in the pelargonidin cinnamic acid complexation-origin of the extraordinary flower color diversity of pharbitis-nil. Journal of the American Chemical Society, 115(8), 3125-3132.

Davis, A. J., \& Mazza, G. (1993). Copigmentation of simple and acylated anthocyanins with colorless phenolic-compounds. Journal of Agricultural and Food Chemistry, 41(5), 716-720.

De Freitas, V. A. P., Glories, Y., \& Laguerre, M. (1998). Incidence of molecular structure in oxidation of grape seed procyanidins. Journal of Agricultural and Food Chemistry, 46(2), 376-382.

Gómez-Míguez, M., González-Manzano, S., Escribano-Bailón, M. T., Heredia, F. J., \& Santos-Buelga, C. (2006). Influence of different phenolic copigments on the color of malvidin 3-glucoside. Journal of Agricultural and Food Chemistry, 54(15), $5422-5429$.

Gonnet, J. F. (1998). Colour effects of copigmentation of anthocyanins revisited - 1 . A colorimetric definition using the CIELAB scale. Food Chemistry, 63(3), 409-415.

Gonzalez-Manzano, S., Rivas-Gonzalo, J. C., \& Santos-Buelga, C. (2004). Extraction of flavan-3-ols from grape seed and skin into wine using simulated maceration. Analytica Chimica Acta, 513(1), 283-289.

Gonzalez-Manzano, S., Santos-Buelga, C., Pérez-Alonso, J. J., Rivas-Gonzalo, J. C., \& Escribano-Bailón, M. T. (2006). Characterization of proanthocyanidin composition in red wines using LC-MS. Journal of Agricultural and Food Chemistry, 54(12), 4326-4332.

González-Manzano, S., Santos-Buelga, C., Dueñas, M., Rivas-Gonzalo, J. C., \& Escribano-Bailón, T. (2008). Colour implications of self-association processes of wine anthocyanins. European Food Research and Technology, 226, 483-490.

Goto, T., \& Kondo, T. (1991). Structure and molecular stacking of anthocyaninsflower color variation. Angewandte Chemie International English Edition, 30, 17-33.

Heredia, F. J., Rivas-Gonzalo, J. C., Vicario, I. M. M., \& Santos-Buelga, C. (1998). Chromatic characterization of anthocyanins from red grapes - I. pH effect. Food Chemistry, 63(4), 491-498.

Heredia, F. J., Álvarez, C., González-Miret, M. L., \& Ramírez, A. (2004). CromaLab ${ }^{\circledR}$, análisis de color, Registro General de la Propiedad Intelectual SE-1052-04 Sevilla, Spain.

Liao, H., Cai, Y., \& Haslam, E. (1992). Polyphenol interactions.6 anthocyaninscopigmentation and color changes in red wines. Journal of the Science of Food and Agriculture, 59(3), 299-305.

Martínez, J. A., Melgosa, M., Pérez, M. M., Hita, E., \& Negueruela, A. I. (2001). Note: Visual and instrumental colour evaluation in red wines. Food Science and Technology International, 7(5), 439-444.

Mirabel, M., Saucier, C., Guerra, C., \& Glories, Y. (1999). Copigmentation in model wine solutions: Ocurrence and relation to wine aging. American Journal of Enology and Viticulture, 50(2), 211-218.

Mistry, T. V., Cai, Y., Lilley, T. H., \& Haslam, E. (1991). Polyphenol interactions. 5. Anthocyanin copigmentation. Journal of the Chemical Society-Perkin Transactions, 2(8), 1287-1296.

Nunez, V., Monagas, M., Gomez-Cordoves, M. C., \& Bartolome, B. (2004). Vitis vinifera L cv. Graciano grapes characterized by its anthocyanin profile. Postharvest Biology and Technology, 31(1), 69-79.

Schwarz, M., Picazo-Bacete, J. J., Winterhalter, P., \& Hermosin-Gutierrez, I. (2005) Effect of copigments and grape cultivar on the color of red wines fermented after the addition of copigments. Journal of Agricultural and Food Chemistry, 53(21), 8372-8381.

Timberlake, C. F., \& Bridle, P. (1976). Interaction between anthocyanins, phenolic compounds and acetaldehyde and their significance in red wine. American Journal of Enology and Viticulture, 27, 97-105. 